\title{
$\begin{array}{ll}\text { Research Square } & \text { Preprints are preliminary reports that have not undergone peer review. } \\ \text { They should not be considered conclusive, used to inform clinical practice, } \\ \text { or referenced by the media as validated information }\end{array}$
}

\section{Long non-coding RNA HOXA-AS2 may serve as a new therapeutic target and promising prognostic market for most of cancers}

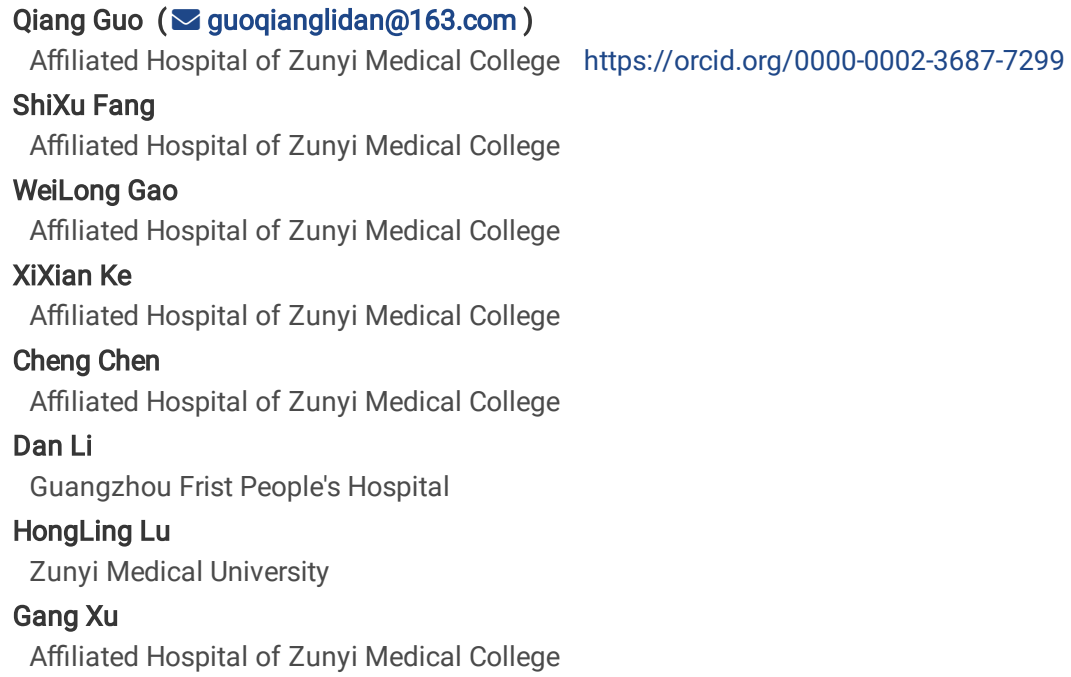




\section{Abstract}

Backgroud: To elucidate the relationship between the expression level of HOXA-AS2 and it's prognostic value of cancer by meta-analysis. Methods: Databases of PubMed, Cochrane Library, Embase, Web of Science, Google Scholar, CNKI and some others were searched systematically. Comprehensively screen according to the inclusion criteria and the exclusion criteria was conducted. The connection between HOXA-AS2 and the prognosis characteristics of patients with various types of cancer was screened by combining odds ratio (OR), Hazard ratios (HR) and 95\% confidence interval (Cl) for the studies collected in this meta-analysis. In addition, we further analyzed the expression of the gene and its potential clinical value in Gene Expression Profiling Interactive Analysis (GEPIA) and the InCAR database. Results: A total of 12 studies consisting 796 patients were included in this research. Compared low HOXA-AS2 expression group, patients with high HOXA-AS2 expression were more likely to get poor overall survival (OS). Moreover, high HOXA-AS2 expression demonstrates advanced TNM stage, earlier lymph node metastasis, distant metastasis and bigger tumor size. But there was no correlation or the correlation was not statistically significant between the expression and the age, sex or the pathological differentiation. In addition, data from the GEPIA and LnCAR databases revealed that increasing HOXA-AS2 expression means bad prognosis in most of cancers. Conclusions: High HOXA-AS2 expression shows worse cancer prognosis in cancer patients, and HOXA-AS2 may be acted as therapeutic target and promising prognostic marker.

\section{Background}

At present, cancer has become one of the major problems imperilling the global public health because of its high morbidity and mortality. Patients in the early stage of cancer can obtain a better long-term prognosis through surgical treatment. However, the tumors of most patients have reached the middle or the late stage at the time of diagnosis, as the result that the prognosis of the middle and late stage patients is still not satisfactory even though they have been treated with active operation, radiotherapy, chemotherapy, endocrine therapy and so on. In recent years, targeted therapy has brought new hope to patients with advanced cancer, and is expected to improve the prognosis of cancer patients. For example, the interaction between opioid binding protein/cell adhesion molecule-like (OPCML) and HER2 destroys the formation of HER2-EGFR heterodimer, which leads to enhanced response of HER2 positive ovarian and breast cancer cells to rapatinib and erlotinib. In addition, increasing the expression of OPCML gene could strengthen the effect of rapatinib in breast cancer patients [1]. Epidermal growth factor receptor variant III (EGFRvIII) is a tumor specific mutation expressed in various types of tumors and it can prevent postoperative recurrence and metastasis through EGFRvIll targeted by CAR-T in patients with lung cancer [2]. Tetra nucleotide targeting regulation of VEGF could also inhibits the growth of non-small cell lung cancer cells [3]. What's more, WM-127, the inhibitor of Survivin, can inhibit the proliferation of hepatocellular carcinoma cells, induce cycle arrest and apoptosis of cancer cells, and at last delay the tumorigenesis of nude mice [4]. Therefore, it is necessary to explore new target molecules and biomarkers as targets for cancer treatment in order to improve the prognosis of tumor patients.

Long-chain non-coding RNA, which does not have the function of protein coding, is a class of RNA with a length of more than 200 nucleotides. Studies had shown that LncRNA was abnormally expressed in a variety of human cancers, and its abnormal expression was significantly related to the proliferation, migration, invasion of cancer cell and so on. For example, the expression of IncRNA PSMG3-AS1 was increased in breast cancer tissues and cells and Interference of PSMG3-AS1 expression can promote the expression of miR-143-3p, resulting in the weakness of proliferation and migration of breast cancer cells [5]. The expression of LINC00467 was up-regulated in HCC tissues and cells., and it could inhibit the proliferation and metastasis of HCC cells and promote apoptosis if LINC00467 was silenced [6]. In addition, the IncRNA TNRC6C-AS1 could increases methylation of STK4 promoter, thus activating the Hippo signaling pathway and promoting the development of thyroid cancer (TC) [7]. It shows that IncRNAs plays a vital role in the occurrence and progression of cancer and is expected to become a new target molecule and biomarker for cancer treatment. The long non-coding RNA HOXA-AS2 is located between the genes of HOXA3 and HOXA4, which is the antisense chain of the homeobox gene A (HOXA). It was found that HOXA-AS2 was expressed in human NB4 promyelocytes and peripheral blood neutrophils, which could be increased after treatment with all-trans retinoic acid (ATRA). And further studies found that HOXA-AS2 could negatively regulated the apoptosis of NB4 cells induced by ATRA [8]. In recent years, it had been reported that HOXA-AS2 was abnormally expressed in colorectal cancer [9-11], acute lymphoblastic leukemia (ALL) and hepatocellular carcinoma [12-14], and was involved in the occurrence and development of tumor. The expression of HOXA-AS2 in colorectal cancer (CRC) was increased and high expression of HOXA-AS2 indicated larger tumor size, later stage, and worse prognosis of patients $[9,10]$. Disturbing the expression of HOXA-AS2 could inhibit the cell cycle G1/S, the proliferation of CRC cells, and induce apoptosis. On the contrary, overexpression of HOXA-AS2 would promote cell migration and invasion by regulating epithelial-interstitial transformation (EMT) $[10,11]$. Moreover, Knocking down HOXA-AS2 can inhibit the proliferation of acute myeloid leukemia (AML) ADR cells and induce apoptosis [12]. And the expression of HOXA-AS2 was also increased in the cell line and patients' samples of prednisone insensitive ALL. HOXA-AS2 can activate the EGFR/Ras/Raf/MEK/ERK signaling pathway and enhance the proliferation ability and inhibit the apoptosis rate of cancer cells by promoting the expression of HOXA3 gene, and then enhance the resistance of glucocorticoid (GC) [13]. The expression of HOXA-AS2 was increased in the tissues and cell lines of hepatic cellular cancer (HCC), knocking down HOXA-AS2 can block the G1 phase, promote the apoptosis, and then inhibit the growth of HCC cells. In addition, HOXAAS2 could leads to migration and invasion of HCC cells by promoting the change of EMT characteristics. These results suggest that HOXA-AS2 plays an important role in the progression of cancer and can be used as a new biomarker and target for tumor therapy [14]. However, the potential clinical value of HOXA-AS2 in the prognosis of tumors is still controversial. Therefore, in this meta-analysis, we will explore the value of HOXA-AS2 in the prognosis and clinicopathological features of cancer for the first time, and also provide new targets and biomarkers for the diagnosis and treatment of cancer patients.

\section{Methods}

\section{Bibliography retrieval}

Search the studies which were published in Pubmed, CochraneLibrary, Embase, WebofScience, GoogleScholar, CNKI and Wanfang databases before February 15, 2019 at home or abroad, and were related to the relationship between HOXA-AS2 and the prognosis and clinicopathological characteristics of tumor 
patients. The Chinese keywords include: "antisense chain of HOXA gene, antisense HOXA gene, tumor, cancer". English search terms include: "LongnoncodingRNA HOXA-AS2" or "LincRNA HOXA-AS2" or "HOXA-AS2" AND "tumor" or "cancer".

\section{Inclusion criteria and exclusion criteria}

Inclusion criteria: 1) The subjects were cancer patients; 2) the expression level of HOXA-AS2 in tissues was detected by quantitative PCR or RT-PCR; 3) there were definite analyses for the relationship between HOXA-AS2 and the clinicopathological features or the OS of the tumor; 4) the patients could be divided into two groups according to the expression level of HOXA-AS2; 5) data on HR, OR and 95\% Cl were provided; 6) the literature was published in English or Chinese. Exclusion criteria: 1) the research species were animals; 2) the data for statistic are incomplete; 3) lack of clinical research data.

\section{Data extraction and quality control}

The literature information was extracted by two researchers independently according to the established screening criteria. The contents of data extraction included: 1) The name of the first author, the years published, the country and region of the researched objects; 2) the characteristics of the objects, including the type of cancer, the total number of cases and the number of patients in different subtypes; 3 ) outcome indicators, including overall survival time, TNM stage, lymph node metastasis, distant metastasis and so on. The HR value and 95\% Cl were obtained by software EngaugeDigitizerversion10.1 (Http://Digitizer. source- forge.net/) for the literatures whose data only include Kaplan-Meier survival curves and lack of HR value, and the estimated HR value and its $95 \% \mathrm{Cl}$ were compared with the survival curve $\mathrm{P}$ value of the original literature. If the information of extracted data was inconsistent, it should be extracted by a third one. 4) the quality evaluation of the literature was independently evaluated by the two researchers according to NOS (The Newcastle-Ottawa Scale), including population selection (the highest score of Selection was 4), comparability (the highest score of Comparability was 2 ), exposure evaluation or result evaluation. NOS adopted the semi-quantitative principle of star system to evaluate the quality of literature. the full score was 9 stars and more than 5 stars were high quality literature.

\section{Validating HOXA-AS2 expression in GEPIA and InCAR databases}

GEPIA is an open database for in-depth analysis of TCGA gene expression data $[15,16]$ LnCAR is a comprehensive and open resource, which has in-depth analysed the expression profile and the prognostic value of the IncRNAs in microarray data. At present, there are 52300 samples in InCAR for differential expression analysis and 12883 samples for survival analysis. The expression of HOXA-AS2 in tumor and its normal samples was analyzed by GEPIA and InCAR databases, and its relationship with staging and prognosis was analyzed at the same time.

\section{Statistical analysis}

The software of Stata 12 and RevMan 5.35 were used for statistical analysis. The HR value and its $95 \% \mathrm{Cl}$ were used as the effect index of survival data, the odds ratio (OR) and it's $95 \% \mathrm{Cl}$ were used as the effect index of clinicopathological characteristics of patients. The HR and its $95 \% \mathrm{Cl}$ should be estimated from the data extracted from the survival curve by EngaugeDigitizer 10.1 if only the Kaplan-Meier survival curve is given in the literature. The heterogeneity of the included results should be judged by $\mathrm{I}^{2}$ quantification. When P-value $\geq 0.05, \mathrm{I}^{2} \leq 50 \%$, the fixed effect model (Mantel-Haenszel method, M-H method) was used for Meta analysis; and when P-value $<0.05$ or $\mathrm{I}^{2}>50 \%$, the heterogeneity was significant, and the random effect model would be used for Meta analysis. The publication bias of the literature was evaluated by Begg's test, and the stability of the results was tested by sensitivity analysis. The difference was thought to be statistically significant if the P-value $<0.05$.

\section{Results}

\section{Incorporate the results of the study}

According to the retrieval strategy, 65 relevant articles were obtained. 16 of them were excluded because of repetitiveness and 19 articles were excluded after reading the title and abstract. Then, through detailed reading of the full text, it is found that 18 articles do not meet the inclusion criteria of this study. Among them, 8 articles did not explore the correlation between the expression of HOXA-AS2 and the prognosis of patients and 10 articles were excluded for incomplete data (Fig. 1). In the end, 12 articles with a total of 796 patients (Table 1) were included in this study. There were 8 types of cancer in these 12 literatures, including 2 cases of lung cancer [17,18], 2 of thyroid cancer [19,20], 1 of bladder cancer [21], 1 of breast cancer [22], 1 of gastric cancer [23], 2 of colorectal cancer [9,10], 1 of osteosarcoma [24] and 2 cases of liver cancer [14,25]. All the 12 articles come from China (Table 1). The Lymph node metastasis, distant metastasis and tumor staging had been confirmed by pathological examination. And all the 12 literature were of high quality according to NOS. 
Table 1

Characteristics of the studies enrolled in the meta-analysis.

\begin{tabular}{|c|c|c|c|c|c|c|c|}
\hline Study & Origin of population & Disease & patients(n) & Stage & Method & Survival analysis & Hazard ratios \\
\hline Cui TJ 2019 & China & NSCLC & 40 & |-II/III-IV & QRT-PCR & KM & $1.11(0.44 \sim 2.8)$ \\
\hline Liu YP 2019 & China & NSCLC & 52 & I-II/III-IV & QRT-PCR & KM & $2.09(1.02 \sim 4.28)$ \\
\hline Xia FD 2018 & China & THCA & 128 & II-IV & QRT-PCR & NA & NA \\
\hline Jiang LF 2018 & China & THCA & 68 & I-II/III-IV & QRT-PCR & KM & $1.98(0.98 \sim 4)$ \\
\hline Wang F 2018 & China & Bladder cancer & 80 & $0-\mathrm{I} / \mathrm{IIIV}$ & QRT-PCR & NA & NA \\
\hline Fang Y 2018 & China & $\mathrm{BC}$ & 38 & 0-II/III-IV & QRT-PCR & KM & $1.57(0.61 \sim 4.04)$ \\
\hline Xie M 2015 & China & $\mathrm{GC}$ & 55 & $|-| I / I I \mid$ & QRT-PCR & $\mathrm{KM}$ & $3.98(1.6 \sim 9.9)$ \\
\hline Li Q 2016 & China & $\mathrm{CRC}$ & 30 & II/III & QRT-PCR & $\mathrm{KM}$ & $4.88(1.69 \sim 14.09)$ \\
\hline Ding J 2017 & China & CRC & 69 & I-II/III-IV & QRT-PCR & NA & NA \\
\hline Wang YH 2018 & China & Osteosarcoma & 66 & NA & QRT-PCR & NA & NA \\
\hline Wang FQ 2016 & China & $\mathrm{HCC}$ & 112 & $|/| I-I I I$ & QRT-PCR & KM & $2.02(1.27 \sim 3.21)$ \\
\hline Zhang Y 2018 & China & $\mathrm{HCC}$ & 58 & III/IV & QRT-PCR & NA & NA \\
\hline
\end{tabular}

Note. NSCLC: non-small cell lung cancer; THCA: thyroid cancer; BC: breast cancer; GC: gastric cancer; CRC: colorectal cancer; HCC: hepatocellular carcinoma; NA: not available; qRT-PCR: quantitative reverse transcription-polymerase chain reaction; KM: Kaplan Meier-plotter.

\section{Association between the expression level of HOXA-AS2 and overall survival time of the patients}

7 literature were enrolled to explore the relationship between HOXA-AS2 expression and the OS in cancer patients. It was found that there was a significant correlation between high expression of HOXA-AS2 and poor OS in tumor patients ( $\mathrm{HR}=2.12,95 \% \mathrm{Cl}=1.61-2.80)$ after combining the effect index (Fig. 2 and Table 2). Because of the small heterogeneity $\left(I^{2}=8 \%, P=0.36\right)$, we adopted the fixed effect model. In this study, we also considered the difference expression of HOXA-AS2 in different tumor tissues. Finally, we found that the $\mathrm{HR}=2.56,95 \% \mathrm{Cl}=1.74-3.77, \mathrm{P}=0.19, \mathrm{I}^{2}=40 \%$ for digestive system and $\mathrm{HR}=1.73,95 \% \mathrm{Cl}=$ $1.16-2.59, P=0.72, I^{2}=0 \%$ for non-digestive system by subgroup analysis according to whether it was the source of digestive system.

Table 2

Subgroup analysis of the pooled HRs with HOXA-AS2 expression in patients with cancer.

\begin{tabular}{|c|c|c|c|c|c|c|c|}
\hline \multirow{3}{*}{$\begin{array}{l}\text { Subgroup analysis } \\
\text { OS }\end{array}$} & \multirow{3}{*}{$\begin{array}{l}\text { No. of studies } \\
7\end{array}$} & \multirow{3}{*}{$\begin{array}{l}\text { No. of patients } \\
395\end{array}$} & \multirow{3}{*}{$\begin{array}{l}\text { Pooled HR } \\
\text { (95\%Cl) } \\
2.12(1.61-2.80)\end{array}$} & \multirow{3}{*}{$\begin{array}{l}P \\
<0.00001\end{array}$} & \multirow{2}{*}{\multicolumn{2}{|c|}{$\begin{array}{l}\text { Heterogeneity } \\
\mathrm{I}^{2}(\%) \text { Pvalue }\end{array}$}} & \multirow{3}{*}{$\begin{array}{l}\text { Model } \\
\text { Fixed }\end{array}$} \\
\hline & & & & & & & \\
\hline & & & & & 8 & 0.36 & \\
\hline Digestive system cancer & 3 & 197 & $2.56(1.74-3.77)$ & $<0.00001$ & 40 & 0.19 & Fixed \\
\hline Non-digestive system cancer & 4 & 198 & $1.73(1.16-2.59)$ & 0.07 & 0 & 0.72 & Fixed \\
\hline \multicolumn{8}{|l|}{ number of patients } \\
\hline more than 60 & 2 & 180 & $2.01(1.36-2.96)$ & 0.0004 & 0 & 0.96 & Fixed \\
\hline less than 60 & 5 & 215 & $2.25(1.51-3.35)$ & $<0.0001$ & 37 & 0.17 & Fixed \\
\hline \multicolumn{8}{|l|}{ Cut-of value } \\
\hline Mean & 3 & 207 & $2.05(1.41-3.00)$ & 0.0002 & 46 & 0.15 & Fixed \\
\hline Median & 4 & 188 & $2.21(1.47-3.32)$ & 0.0001 & 0 & 0.43 & Fixed \\
\hline \multicolumn{8}{|l|}{ Quality scores } \\
\hline Score $=9$ & 5 & 327 & $2.05(1.51-2.77)$ & $<0.00001$ & 0 & 0.44 & Fixed \\
\hline Score $<9$ & 2 & 68 & $2.70(0.89-8.18)$ & 0.08 & 59 & 0.12 & Random \\
\hline
\end{tabular}

\section{Association between expression level of HOXA-AS2 and TNM staging}

The relationship between the expression of HOXA-AS2 and TNM staging was reported in 11 of the 12 literature (Fig. 3A and Table 3). Because of the small heterogeneity $\left(P=0.167, I^{2}=29.2 \%\right)$, we adopted the fixed effect model in the same way, and the combined OR value and its $95 \% \mathrm{Cl}$ were 4.40 and $3.16-6.14$ $\left(P=0.22, I^{2}=32 \%\right)$, respectively. In addition, Subgroup analysis based on cancer type showed that high expression of HOXA-AS2 was significantly correlated 
with TNM staging in respiratory system $(\mathrm{OR}=6.81,95 \% \mathrm{Cl}=2.38-19.46)$, digestive system $(\mathrm{OR}=3.56,95 \% \mathrm{Cl}=2.2-5.75)$, and other systems $(\mathrm{OR}=5.01,95 \% \mathrm{Cl}$ $=2.98-8.41$ ), indicating that patients with high expression were more likely to get advanced stage of cancer.

Table 3

Pool effects of Clinicopathologic characteristics in cancer patients with abnormal HOXA-AS2 expression.

\begin{tabular}{|c|c|c|c|c|c|c|}
\hline \multirow{3}{*}{$\begin{array}{l}\text { Clinicopathological characteristics } \\
\text { Age }\end{array}$} & \multirow{3}{*}{$\begin{array}{l}\text { No. of studies } \\
11\end{array}$} & \multirow{3}{*}{$\begin{array}{l}\text { No. of patients } \\
758\end{array}$} & \multirow{3}{*}{$\begin{array}{l}\text { Pooled HR } \\
(95 \% \mathrm{Cl}) \\
1.11(0.83-1.48)\end{array}$} & \multirow{3}{*}{$\begin{array}{l}P \\
0.562\end{array}$} & \multirow{2}{*}{\multicolumn{2}{|c|}{$\begin{array}{l}\text { Heterogeneity } \\
\mathrm{I}^{2}(\%) \text { P-value }\end{array}$}} \\
\hline & & & & & & \\
\hline & & & & & 0 & 0.494 \\
\hline Gender & 11 & 758 & $1.11(0.81-1.52)$ & 0.469 & 9.1 & 0.355 \\
\hline TNM stage & 11 & 758 & $4.4(3.16-6.14)$ & 0.00001 & 29.2 & 0.167 \\
\hline Digestive system & 5 & 324 & $3.56(2.20-5.75)$ & 0.0001 & 14.5 & 0.322 \\
\hline Respiratory system & 2 & 92 & $6.81(2.38-19.46)$ & 0.0001 & 65.9 & 0.087 \\
\hline Other system malignancy & 4 & 262 & $5.01(2.98-8.41)$ & 0.0001 & 46.6 & 0.131 \\
\hline LNM & 7 & 492 & $6.20(4.01-9.59)$ & 0.0001 & 34.5 & 0.164 \\
\hline Digestive system & 2 & 124 & $4.49(1.93-10.42)$ & 0.0002 & 0 & 0.667 \\
\hline Respiratory system & 2 & 92 & $5.52(1.98-15.40)$ & 0.001 & 49 & 0.161 \\
\hline Other system malignancy & 3 & 276 & 7.49 (4.14-13.55) & 0.0001 & 70.3 & 0.034 \\
\hline Tumor size (big vs total) & 8 & 560 & $1.56(1.25-1.95)$ & 0.020 & 66.0 & 0.000 \\
\hline Digestive system & 3 & 247 & $4.86(2.74-8.61)$ & 0.005 & 0 & 0.825 \\
\hline Other system malignancy & 5 & 324 & $1.73(1.11-2.71)$ & 0.000 & 0 & 0.729 \\
\hline Histological grade & 3 & 258 & $1.34(0.78-2.28)$ & 0.082 & 0 & 0.948 \\
\hline Distant metastases & 3 & 164 & $3.78(1.92-7.47)$ & 0.001 & 0 & 0.655 \\
\hline Invasion depth(T3 + T4/T1 + T2) & 3 & 261 & $2.12(1.28-3.53)$ & 0.02 & 14.1 & 0.312 \\
\hline
\end{tabular}

\section{Association between expression level of HOXA-AS2 and lymph node metastasis}

The relationship between the expression of HOXA-AS2 and lymph node metastasis was reported in 7 of the 12 literature (Fig. 3B and Table 3). Because of the small heterogeneity $\left(P=0.164, I^{2}=34.5 \%\right)$, the fixed effect model was used again, and the combination of OR and $95 \% \mathrm{Cl}$ showed that the high expression group was more likely to have metastasis $(\mathrm{OR}=6.2,95 \% \mathrm{Cl}=4.01-9.59)$ than the low expression group. According to the subgroup analysis of cancer types, it was found that high expression of HOXA-AS2 was significantly correlated with lymph node metastasis in tumors of respiratory system $(\mathrm{OR}=5.52,95 \% \mathrm{Cl}=$ $1.98-15.40)$, digestive system $(\mathrm{OR}=4.49,95 \% \mathrm{Cl}=1.93-10.42)$ and other systems $(\mathrm{OR}=7.49,95 \% \mathrm{Cl}=4.14-13.55)$. These results suggest that patients with high expression of HOXA-AS2 are more likely to develop lymph node metastasis.

\section{Association between expression level of HOXA-AS2 and distant metastasis}

The relationship between HOXA-AS2 expression and distant metastasis (DM) of tumors was reported in 3 of the 12 literature (Fig. $3 \mathrm{C}$ and Table 3 ). Because of the low heterogeneity $\left(P=0.655, I^{2}=0\right)$, we used the fixed effect model. The combined OR and its $95 \% \mathrm{Cl}$ was 3.78 and $1.922-7.47$, respectively, indicating that patients with high expression of HOXA-AS2 were more likely to have distant metastasis of cancer cells.

\section{Association between expression level of HOXA-AS2 and other clinicopathological parameters}

This study further investigated the correlation between HOXA-AS2 expression level and age, sex, pathological grade, depth of invasion and tumor size of patients. We found that high expression level of HOXA-AS2 was positively correlated with the depth of the tumor size $(\mathrm{OR}=2.58,95 \% \mathrm{Cl}=1.82-3.64)$ and invasion ( $\mathrm{HR}=2.12,95 \% \mathrm{Cl}=1.28-3.53)$ (Fig. 4). However, the level of $\mathrm{HOXA}-\mathrm{AS} 2$ expression was not correlated with the age $(\mathrm{OR}=1.11,95 \% \mathrm{Cl}=0.83-1.48)$, the sex $(\mathrm{OR}=1.11,95 \% \mathrm{Cl}=0.81-1.52)$ and the histological grade $(\mathrm{OR}=1.34,95 \% \mathrm{Cl}=0.78-2.28)$ of patients.

\section{Publication bias and sensitivity Analysis}

In this study, Stata 12.0 software was used to continuously delete the results of each included literature, so as to analyze the sensitivity of the included literature (Fig. 5A). We found that the results of individual studies had no significant impact on the overall results, which meant that each of the included results was consistent with the overall results. We further analyzed the publication bias of each index according to the Begg test. As shown in Fig. 5B, OS (Pr > $|z|=0.245)$ have less publication bias. TNM phase $(\operatorname{Pr}>|z|=0), \operatorname{LNM}(\operatorname{Pr}>|z|=0.493), D M(\operatorname{Pr}>|z|=0.245)$, histological grade $(\operatorname{Pr}>|z|=0.245)$ and tumor size $(\operatorname{Pr}>|z|=0.497)$ have the same result. Thus, we believe that the results of this study are credible.

Clinical value of HOXA-AS2 in GEPIA and InCAR datasets 
In order to further verify our results, GEPIA and InCAR database were applied to analyze the expression and prognosis of HOXA-AS2 in various cancers, and to explore the possible mechanism of its effect on cancer progression. As shown in Fig. 6A, the expression of HOXA-AS2 is decreased in lung adenocarcinoma (LUAD), lung squamous cell carcinoma (LUSC), thyroid carcinoma (THCA), bladder cancer (BLCA), breast invasive carcinoma (BRCA), colon adenocarcinoma (COAD), osteosarcoma (SARC) and liver cancer (LIHC). And the expression was increased in gastric cancer (STAD). The violin chart showed that the expression level of HOXA-AS2 was significantly correlated with the clinical stage of human cancer (Fig. 6B). In addition,we divided the cases into high and low expression groups according to the median value of HOXA-AS2, and the results showed that up-regulation of HOXA-AS2 expression predicted poor OS and DFS in cancer patients (Fig. 6C-D) by combining the data of HOXA-AS2, OS and DFS of all tumors in the GEPIA dataset. Moreover, we had confirmed in the InCAR database that the expression of HOXA-AS2 was decreased in lung cancer, non-small cell lung cancer, lung adenocarcinoma, lung squamous cell carcinoma, large cell lung cancer, and breast cancer (Table 4, Adjust $\mathrm{P}<0.05$ ). And the expression level of HOXA-AS2 was correlated with the prognosis of bladder cancer, breast cancer, colorectal cancer and gastric cancer (Fig. 7). The ceRNA network signal diagram showed that the HOXA-AS2 regulates the expression of mRNA through miRNA (miR-372-3p; miR-373-3p; miR-302a-3p; miR-519d-3p; miR-106b-5p; miR-520b; miR-302e; miR-520e; miR-106a-5p; miR302d-3p; miR-302c-3p; miR-302b-3p; miR-20a-5p; miR-520d-3p; miR-17-5p; miR-93-5p; miR-20b-5p; miR-520c-3p and miR-520a-3p) so as to mediate the occurrence and development of tumors (Fig. S1 and Table 5). We also found that HOXA-AS2 was associated with cancer, MAPK pathway, NOD receptor pathway, Ca ion signaling pathway through KEGG (Fig. 8). These results are consistent with the results of this meta-analysis, indicating that HOXA-AS2 has a very important value in tumor, and is expected to become a new target and biomarker for cancer treatment. 
Table 4

Expression of HOXA-AS2 in various cancers.

\begin{tabular}{|c|c|c|c|c|c|}
\hline Analysis ID & Tumor & Gene & $\mathbf{N}$ & LogFC & Adjusted P Value \\
\hline LC_S113 & Lung cancer & HOXA-AS2 & 50 & -0.5953 & 0.0000 \\
\hline LC_S13 & Lung cancer & HOXA-AS2 & 16 & -2.2631 & 0.0003 \\
\hline LC_S3 & Non-small cell lung cancer & HOXA-AS2 & 66 & -0.4817 & 0.0000 \\
\hline LC_S243 & Non-small cell lung cance & HOXA-AS2 & 117 & -0.3702 & 0.0000 \\
\hline LC_S216 & Lung adenocarcinoma & HOXA-AS2 & 50 & -0.5703 & 0.0000 \\
\hline LC_S223 & Lung adenocarcinoma & HOXA-AS2 & 88 & -2.4378 & 0.0000 \\
\hline LC_S257 & Lung adenocarcinoma & HOXA-AS2 & 194 & -0.6801 & 0.0000 \\
\hline LC_S263 & Lung adenocarcinoma & HOXA-AS2 & 110 & -0.4703 & 0.0000 \\
\hline LC_S72 & Lung adenocarcinoma & HOXA-AS2 & 166 & 0.3201 & 0.0089 \\
\hline LC_S130 & Lung adenocarcinoma & HOXA-AS2 & 12 & 0.4931 & 0.0180 \\
\hline LC_S218 & Squamous cell lung cancer & HOXA-AS2 & 20 & -0.4879 & 0.0034 \\
\hline LC_P19 & Large-cell lung carcinoma & HOXA-AS2 & 19 & -2.0295 & 0.0035 \\
\hline LC_S244 & Lung carcinoid tumor & HOXA-AS2 & 13 & -0.7596 & 0.0013 \\
\hline LC_S187 & Small cell lung cancer & HOXA-AS2 & 68 & -0.2088 & 0.0015 \\
\hline BC_S32 & Urothelial carcinoma & HOXA-AS2 & 17 & -0.5522 & 0.0182 \\
\hline BC_S48 & Urothelial carcinoma & HOXA-AS2 & 18 & -0.6035 & 0.0263 \\
\hline BC_S4 & Bladder cancer & HOXA-AS2 & 8 & -0.5886 & 0.0005 \\
\hline BT_S497/BT_S502 & Breast cancer & HOXA-AS2 & 164 & -0.4866 & 0.0000 \\
\hline BT_S217 & Breast cancer & HOXA-AS2 & 53 & -0.3899 & 0.0002 \\
\hline BT_S292 & Breast cancer & HOXA-AS2 & 108 & -0.2791 & 0.0002 \\
\hline BT_S534 & Breast cancer & HOXA-AS2 & 94 & -0.4685 & 0.0007 \\
\hline BT_S327 & Breast cancer & HOXA-AS2 & 45 & -1.7424 & 0.0049 \\
\hline BT_S582 & Breast cancer & HOXA-AS2 & 40 & -0.2219 & 0.0133 \\
\hline BT_S302 & Breast cancer & HOXA-AS2 & 121 & -0.1562 & 0.0277 \\
\hline BT_S290 & Breast cancer & HOXA-AS2 & 47 & -0.2142 & 0.0382 \\
\hline BT_S58 & Breast cancer & HOXA-AS2 & 6 & -1.0087 & 0.0423 \\
\hline BT_S581 & Basal-like breast cancer & HOXA-AS2 & 37 & -0.2664 & 0.0032 \\
\hline BT_S498/BT_S503 & HER2 breast cancer & HOXA-AS2 & 50 & -0.4811 & 0.0000 \\
\hline BT_S499/BT_S504 & Luminal A breast cancer & HOXA-AS2 & 40 & -0.3552 & 0.0000 \\
\hline BT_S500/BT_S505 & Luminal B breast cancer & HOXA-AS2 & 41 & -0.4617 & 0.0000 \\
\hline BT_S501/BT_S506 & Triple-negative breast cancer & HOXA-AS2 & 66 & -0.5733 & 0.0000 \\
\hline BT_P20 & Invasive ductal carcinoma & HOXA-AS2 & 73 & -0.6308 & 0.0000 \\
\hline BT_S483 & Invasive ductal carcinoma & HOXA-AS2 & 58 & -0.4307 & 0.0004 \\
\hline BT_S3 & Invasive ductal carcinoma & HOXA-AS2 & 185 & -0.0821 & 0.0098 \\
\hline BT_S5 & Invasive ductal carcinoma & HOXA-AS2 & 44 & -0.1427 & 0.0166 \\
\hline CR_S107 & Colorectal cancer & HOXA-AS2 & 148 & -0.1220 & 0.0016 \\
\hline CR_S1 & Colorectal cancer & HOXA-AS2 & 12 & -1.2099 & 0.0041 \\
\hline CR_S14 & Colorectal cancer & HOXA-AS2 & 136 & -0.2165 & 0.0100 \\
\hline CR_S128 & Colorectal cancer & HOXA-AS2 & 111 & -0.1036 & 0.0024 \\
\hline CR_S28 & Colon cancer & HOXA-AS2 & 13 & -0.7113 & 0.0004 \\
\hline CR_S42 & Colon cancer & HOXA-AS2 & 64 & -0.4134 & 0.0005 \\
\hline
\end{tabular}




\begin{tabular}{|llllll|}
\hline Analysis ID & Tumor & Gene & N & LogFC & Adjusted P Value \\
\hline CR_S193 & Colon cancer & HOXA-AS2 & 37 & -0.3719 & 0.0021 \\
\hline GT_S5 & Gastric cancer & HOXA-AS2 & 132 & 0.2505 & 0.0017 \\
\hline GT_S31 & Gastric cancer & HOXA-AS2 & 64 & 0.9784 & 0.0029 \\
\hline GT_S92 & Gastric cancer & HOXA-AS2 & 69 & 0.1060 & 0.0081 \\
\hline GT_S51 & Gastric cancer & HOXA-AS2 & 6 & 0.9947 & 0.0105 \\
\hline GT_S13 & Gastric adenocarcinoma & HOXA-AS2 & 20 & 0.4815 & 0.0138 \\
\hline GT_S88/GT_S89 & Gastric adenocarcinoma & HOXA-AS2 & 44 & 0.0897 & 0.0180 \\
\hline GT_S73 & Gastric adenocarcinoma & HOXA-AS2 & 40 & 0.0813 & 0.0230 \\
\hline LI_S151 & Hepatoblastoma & HOXA-AS2 & 8 & 1.2975 & 0.0132 \\
\hline LI_S239 & Hepatocellular carcinoma & HOXA-AS2 & 84 & 0.7407 & 0.0000 \\
\hline LI_S246 & Hepatocellular carcinoma & HOXA-AS2 & 167 & 0.4750 & 0.0001 \\
\hline LI_S265 & Hepatocellular carcinoma & HOXA-AS2 & 140 & 0.1328 & 0.0001 \\
\hline LI_S456 & Hepatocellular carcinoma & HOXA-AS2 & 433 & 0.0836 & 0.0001 \\
\hline LI_S240 & Hepatocellular carcinoma & HOXA-AS2 & 119 & -0.1508 & 0.0004 \\
\hline
\end{tabular}




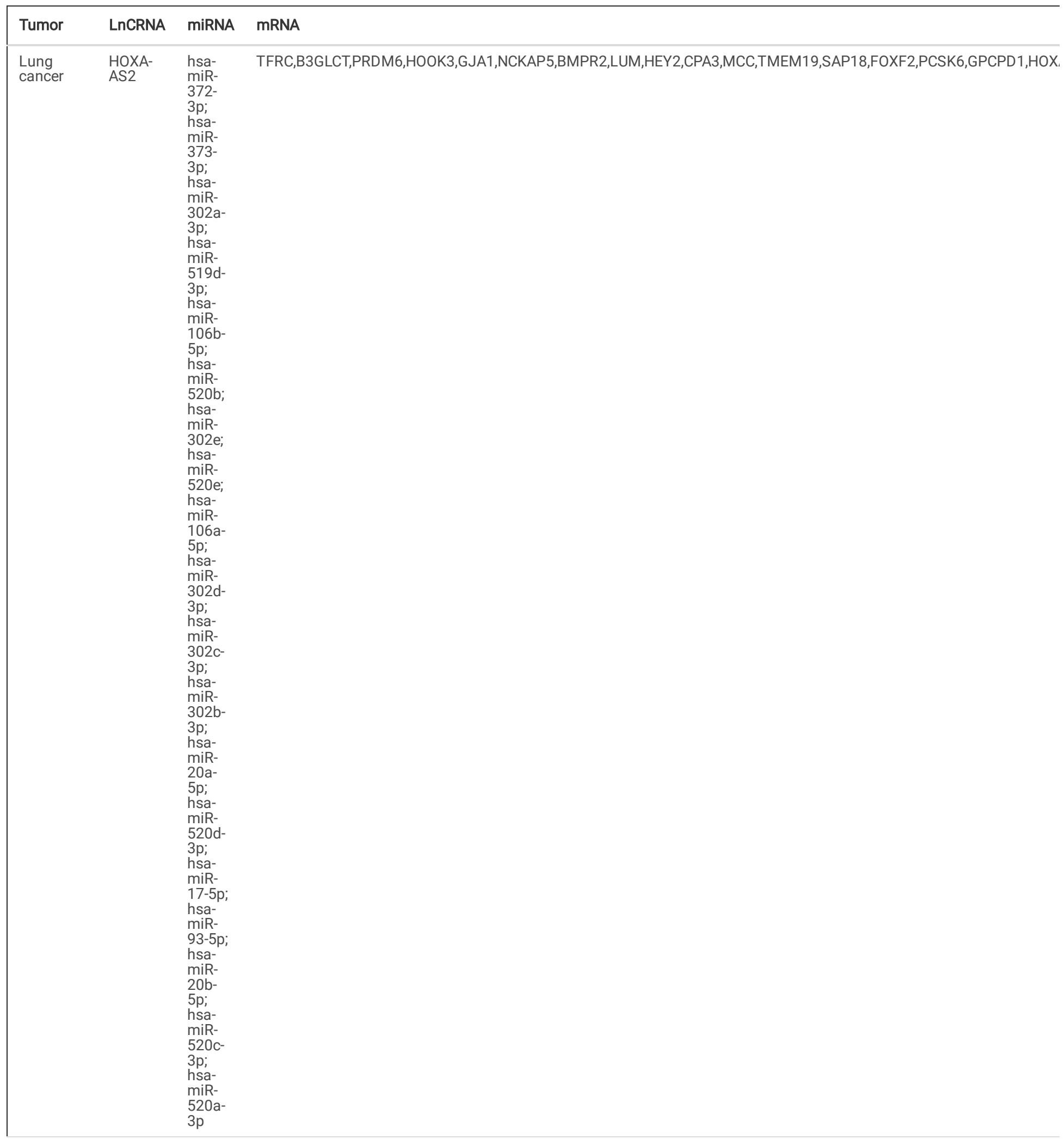




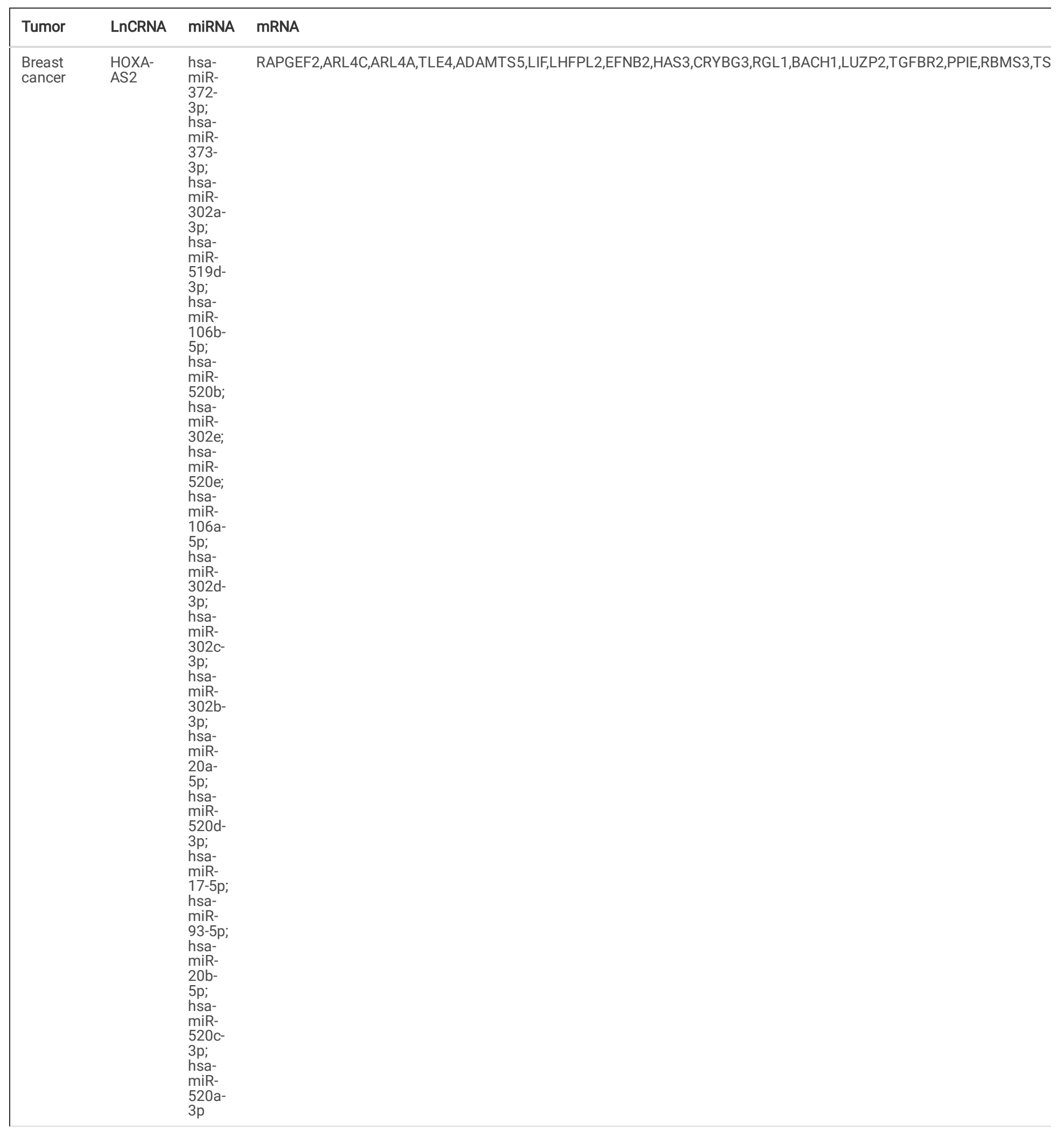




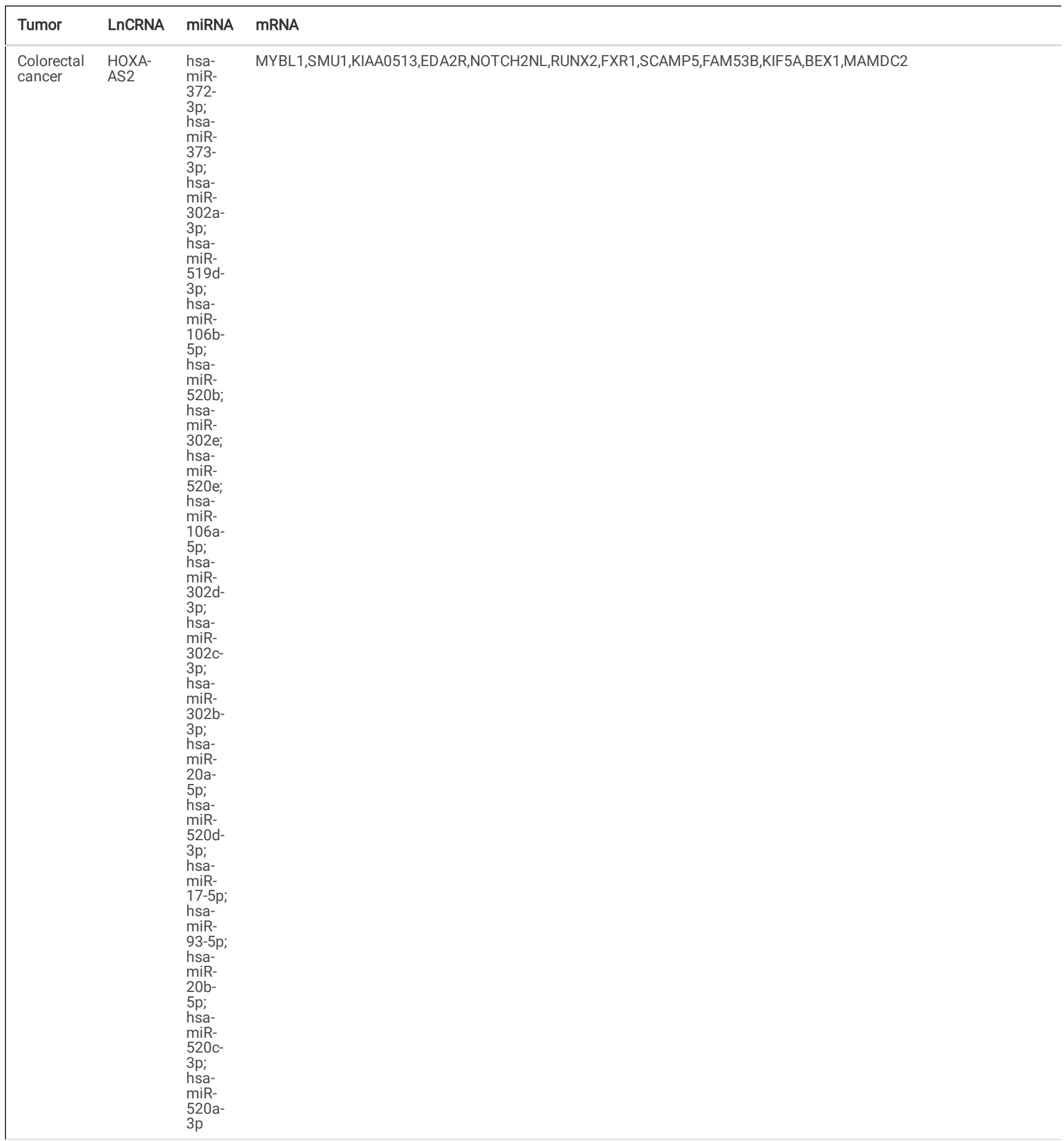




\begin{tabular}{|c|c|c|c|}
\hline Tumor & LnCRNA & miRNA & mRNA \\
\hline $\begin{array}{l}\text { Liver } \\
\text { Cancer }\end{array}$ & $\begin{array}{l}\text { HOXA- } \\
\text { AS2 }\end{array}$ & 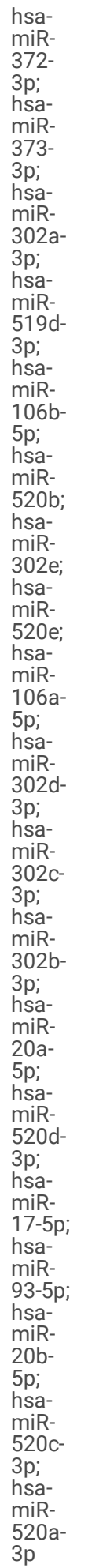 & HNRNPA2B1,WDR48,ZNF280C,HADHA,EFNB2,SPRED1,SRPX,HSD17B11,CCAR1,FOXC1,CPOX,GPR180,BLCAP,RAD51AP1, \\
\hline Figure & & & \\
\hline
\end{tabular}

\section{Discussion}

With the continuous breakthrough of the genome, high-throughput sequencing and the rapid development of cancer genome map, growing evidences have manifested that IncRNA is involved in the process of a variety of human diseases. At present, IncRNA has been found to express abnormally in a variety of cancers and is involved in the occurrence and development of cancer as an oncogene or tumor suppressor gene. For example, the expression of LINC00271 was down-regulated in adrenocortical carcinoma, and it tend to develop a poor prognosis for patients [26]. The expression of IncRNA FALEC in plasma of patients with cervical cancer was significantly up-regulated, and was significantly correlated with the tumor size, FIGO stage and lymph node metastasis. 
What's more, overexpression of FALEC could promote the proliferation and invasion of Hela cells [27]. These results suggest that InCRNA provides a new prospect for the treatment of human cancer.

This meta-analysis found that increased HOXA-AS2 expression was significantly correlated with poor OS in tumor patients without significant publication bias. After further analyses of the association between HOXA-AS2 expression and the clinicopathological features of tumor patients, we found that high expression of HOXA-AS2 was correlated with lymph node metastasis, distant metastasis, advanced staging and larger tumor diameter, the same as the correlation between the prognosis and HOXA-AS2. Unfortunately, there is no evidence showed that high expression level of HOXA-AS2 was associated with the age, sex and pathological grade of tumor patients in this study. These results suggested that HOXA-AS2 is involved in the progression of cancer as an oncogene and is expected to be a new target and prognostic biomarker for cancer therapy. In addition, we confirmed the abnormal expression of HOXA-AS2 in LUAD, LUSC, THCA and other cancers in GEPIA data set, and that the expression level of HOXA-AS2 was correlated with the clinical stage and adverse OS of human cancers. Therefore, we speculate that HOXA-AS2 is an independent risk factor for cancer prognosis and is expected to be a new target and biomarker for cancer treatment.

Through the InCAR database, we found that HOXA-AS2 can regulate the expression of mRNA through miR-372-3p, miR-373-3p, miR-302a-3p, miR-519d-3p, miR-106b-5p, miR-520b, miR-302e, miR-520e, miR-106a-5p, miR-302d-3p, miR-302c-3p, MiR-302b-3p, miR-20a-5p, miR-520d-3p, miR-17-5p, miR-93-5p, miR-20b$5 p$, miR-520c-3p and miR-520a-3p, and further participate in the occurrence and development of tumor. At present, studies have confirmed that long-chain noncoding RNAHOXA-AS2 can take part in the progression of AML, thyroid papillary carcinoma, HCC and breast cancer throuh target regulating of miR-520c-3p $[12,14,19,22]$. In addition, HOXA-AS2 can promote the malignant progression of non-small cell lung cancer, thyroid papillary carcinoma and liver cancer through miRNA-216a-5p, miR-520a-3p, miR-15a-5p and miR-125b [17,18,20,21]. And the KEGG showed that HOXA-AS2 was associated with cancer, MAPK pathway, NOD receptor pathway and Ca ion signaling pathway. For instance, LTB4 stimulated the growth of human pancreatic cancer cells through MAPK and PI-3 kinase pathways [28]. And HOXA-AS2 can reduce glucocorticoid sensitivity in patients with ALL leukemia by regulating the HOXA3/EGFR /Ras/Raf/MEK/ERK pathway [13]. It further showed the importance of HOXA-AS2 in the occurrence and development of tumor.

However, there are also some shortcomings in this study. First, all the studies included in this study were from China, although the prognostic value of HOXAAS2 in GEPIA and InCAR databases was consistent with the results of this meta-analysis, the results of this study may only be applicable to Chinese or Asian populations. Second, most of the literature collected in this study are small sample studies, thus there may be the possibility of systematic bias, more large sample studies are needed to support our conclusion. Third, some negative or contrary conclusions may not be published, so it may cause publishing bias; Fourth, when extracting the HR value from Kaplan-Meier survival curve, there may be extraction or calculation deviation. This meta-analysis confirmed that the high expression level of HOXA-AS2 was associated with the poor prognosis of a variety of cancers, and encouraged researchers to further explore the clinical value and molecular regulation mechanism of HOXA-AS2 at the tissue and cellular level.

\section{Conclusions}

The increased expression of HOXA-AS2 is associated with the poor prognosis of cancer patients. HOXA-AS2 can be used as a new target and biomarker for cancer treatment, which is beneficial to the early diagnosis for cancer patients and to the identification of the patients with poor prognosis.

\section{Abbreviations}

OR: odds ratio; HR: Hazard ratios; Cl: confidence interval『GEPIA: Gene Expression Profiling Interactive Analysis $₫$ OS: overall survival; OPCM: opioid binding protein/cell adhesion molecule-like区EGFRvIII: Epidermal growth factor receptor variant IIIXTC: thyroid cancer囚HOXA: homeobox gene A囚ALL: acute lymphoblastic leukemia $\triangle C R C$ : colorectal cancer囚EMT: epithelial-interstitial transformation $\triangle A M L$ : acute myeloid leukemia『HCC: hepatic cellular cancer.

\section{Declarations}

\section{Ethics approval and consent to participate}

This study is not need any ethics committee's agreement, and does not violate the rights of other persons or institutions.

\section{Consent for publication}

All authors agree to publish the manuscript

\section{Availability of data and material}

The datasets generated for this study are available on request to the corresponding author.

\section{Competing interests}

The authors declare that there is no conflict of interest regarding the publication of this paper.

\section{Funding}

This work is supported by Grants from the National Natural Science Foundation of China (No. 81960532). And LHL, GWL, KXX, CC, LD specify this metaanalysis standard to assist in screening the literature. GWL and KXX independently evaluated the included literature. 


\section{Authors' contributions}

This article was finished by all the authors. LHL, XG and GQ conceived the research topic, made the research plan and directed the implementation of the whole research. GQ and FSX drafted the manuscript together and processed the data. LHL, GWL, KXX, CC and LD specify this meta-analysis standard to assist in screening the literature. GWL and KXX independently evaluated the included literature. LD reevaluated the disputed literature. All authors read and approved the final manuscript.

\section{Acknowledgements}

Not applicable

All authors read and approved the final manuscript. The authors declare that there is no conflict of interest regarding the publication of this paper.

\section{References}

1. Han X, Guo Y, Gao H, et al. Estimating the spatial distribution of environmental suitability for female lung cancer mortality in China based on a novel statistical method. Environ Sci Pollut Res Int. 2019; 26: 10083-10096.

2. Zhang Z, Jiang J, Wu X, et al. Chimeric antigen receptor T cell targeting EGFRvIll for metastatic lung cancer therapy. Front Med. 2019; 13: 57-68.

3. Muench D, Rezzoug F, Thomas S, D et al. Quadruplex-forming oligonucleotide targeted to the VEGF promoter inhibits growth of non-small cell lung cancer cells. PLoS ONE. 2019; 14: e0211046.

4. Yin H, Que R, Liu C, et al. Survivin-targeted drug screening platform identifies a matrine derivative WM-127 as a potential therapeutics against hepatocellular carcinoma. Cancer Lett. 2018; 425: 54-64.

5. Cui Y, Fan Y, Zhao G, et al. Novel IncRNA PSMG3-AS1 functions as a miR-143-3p sponge to increase the proliferation and migration of breast cancer cells. Oncol Rep. 2020; 43(1):229-239.

6. Jiang W, Cheng X, Wang T, et al. LINC00467 promotes cell proliferation and metastasis by binding with IGF2BP3 to enhance the mRNA stability of TRAF5 in hepatocellular carcinoma. J Gene Med. 2019; undefined: e3134.

7. Peng X, Ji C, Tan L, et al. Long non-coding RNA TNRC6C-AS1 promotes methylation of STK4 to inhibit thyroid carcinoma cell apoptosis and autophagy via Hippo signalling pathway. J Cell Mol Med. 2020; 24: 304-316.

8. Zhao H, Zhang X, Frazão $\mathrm{J}$ B, et al. HOX antisense lincRNA HOXA-AS2 is an apoptosis repressor in all trans retinoic acid treated NB4 promyelocytic leukemia cells. J Cell Biochem. 2013; 114: 2375-83.

9. Li Q, Dai Y, Wang F, et al. Differentially expressed long non-coding RNAs and the prognostic potential in colorectal cancer. Neoplasma. 2016; 63: 977-983.

10. Ding J, Xie M, Lian Y, et al. Long noncoding RNA HOXA-AS2 represses P21 and KLF2 expression transcription by binding with EZH2, LSD1 in colorectal cancer. Oncogenesis. 2017; 6: e288.

11. Tong G, Wu X, Cheng B, et al. Knockdown of HOXA-AS2 suppresses proliferation and induces apoptosis in colorectal cancer. Am J Transl Res. 2017; 9 : 4545-4552.

12. Dong X, Fang Z, Yu M, et al. Knockdown of Long Noncoding RNA HOXA-AS2 Suppresses Chemoresistance of Acute Myeloid Leukemia via the miR-520c3p/S100A4 Axis. Cell Physiol Biochem. 2018; 51: 886-896.

13. Zhao Q, Zhao S, Li J, et al. TCF7L2 activated HOXA-AS2 decreased the glucocorticoid sensitivity in acute lymphoblastic leukemia through regulating HOXA3/EGFR/Ras/Raf /MEK/ERK pathway. Biomed Pharmacother. 2019; 109: 1640-1649.

14. Zhang Y, Xu J, Zhang S, et al. HOXA-AS2 Promotes Proliferation and Induces Epithelial-Mesenchymal Transition via the miR-520c-3p/GPC3 Axis in Hepatocellular Carcinoma. Cell Physiol Biochem. 2018; 50: 2124-2138.

15. Tang Z, Li C, Kang B, et al. GEPIA: a web server for cancer and normal gene expression profiling and interactive analyses. Nucleic Acids Res. 2017; 45 : W98-W102.

16. Zheng Y, Xu Q, Liu M, et al. InCAR: A Comprehensive Resource for IncRNAs from Cancer Arrays. Cancer Res. 2019; 79: $2076-2083$.

17. Cui TJ, Lin GS, Dai YM, et al. LncRNA HOXA-AS2 regulates microRNA-216a-5p to promote malignant progression of non-small cell lung cancer. Eur Rev Med Pharmacol Sci. 2019; 23: 264-273.

18. Liu Y, Lin X, Zhou S, et al. Long noncoding RNA HOXA-AS2 promotes non-small cell lung cancer progression by regulating miR-520a-3p. Biosci Rep. 2019; 39: undefined.

19. Xia F, Chen Y, Jiang B, et al. Long Noncoding RNA HOXA-AS2 Promotes Papillary Thyroid Cancer Progression by Regulating miR-520c-3p/S100A4 Pathway. Cell Physiol Biochem. 2018; 50: 1659-1672.

20. Jiang L, Wu Z, Meng X, et al. LncRNA HOXA-AS2 Facilitates Tumorigenesis and Progression of Papillary Thyroid Cancer by Modulating the miR-15a5p/HOXA3 Axis. Hum Gene Ther. 2019; 30: 618-631.

21. Wang F, Wu D, Chen J, et al. Long non-coding RNA HOXA-AS2 promotes the migration, invasion and stemness of bladder cancer via regulating miR125b/Smad2 axis. Exp Cell Res. 2019; 375: 1-10.

22. Fang $\mathrm{Y}$, Wang J, Wu F, et al. Long non-coding RNA HOXA-AS2 promotes proliferation and invasion of breast cancer by acting as a miR-520c-3p sponge. Oncotarget. 2017; 8: 46090-46103. 
23. Xie M, Sun M, Zhu Y, et al. Long noncoding RNA HOXA-AS2 promotes gastric cancer proliferation by epigenetically silencing P21/PLK3/DDIT3 expression. Oncotarget. 2015; 6: 33587-601.

24. Wang Y, Zhang R, Cheng G, et al. Long non-coding RNA HOXA-AS2 promotes migration and invasion by acting as a ceRNA of miR-520c-3p in osteosarcoma cells. Cell Cycle. 2018; 17: 1637-1648.

25. Wang F, Yang H, Deng Z, et al. HOX Antisense lincRNA HOXA-AS2 Promotes Tumorigenesis of Hepatocellular Carcinoma. Cell Physiol Biochem. 2016; 40: 287-296.

26. Buishand Floryne O, Liu-Chittenden Y, Fan Y, et al. Adrenocortical tumors have a distinct, long, non-coding RNA expression profile and LINC00271 is downregulated in malignancy. Surgery. 2020; 167: 224-232.

27. Naizhaer G, Kuerban A, Meilipa R, et al. Up-regulation of IncRNA FALEC indicates prognosis and diagnosis values in cervical cancer. Pathol Res Pract. 2019; 215: 152495.

28. Tong WG, Ding XZ, Talamonti MS, et al. LTB4 stimulates growth of human pancreatic cancer cells via MAPK and PI-3 kinase pathways. Biochem Biophys Res Commun. 2005; 335: 949-56.

\section{Supplementary File Legend}

Fig. S1 The targeted regulatory relationship of HOXA-AS2-miRNA-mRNA in various cancers.

\section{Figures}

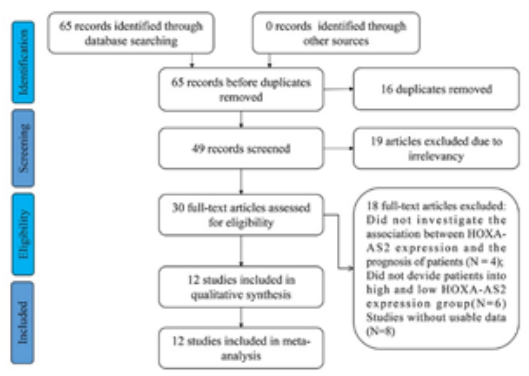

\section{Figure 1}

Flow diagram of the study search and selection process.

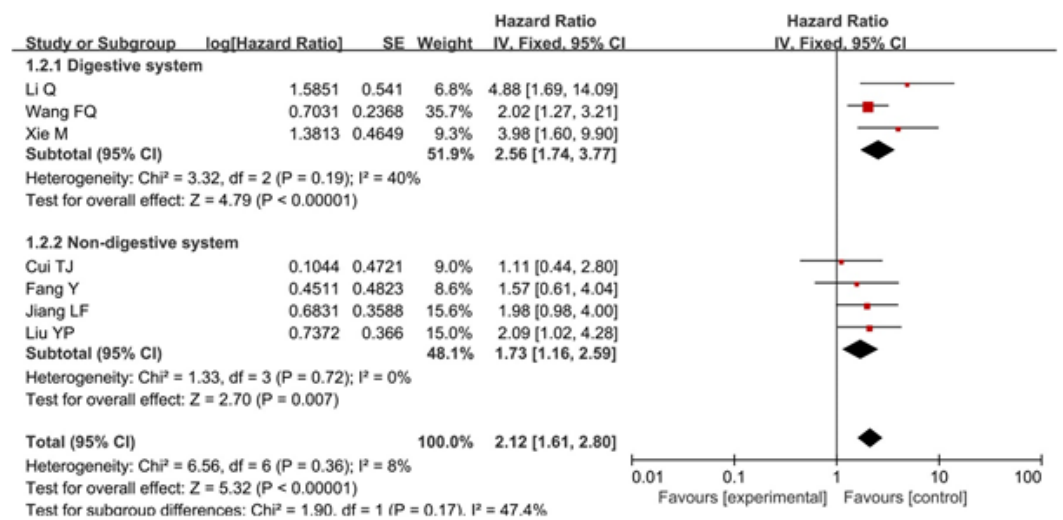

\section{Figure 2}

Forest plot for the association between SNHG1 expression levels with overall survival (OS). 


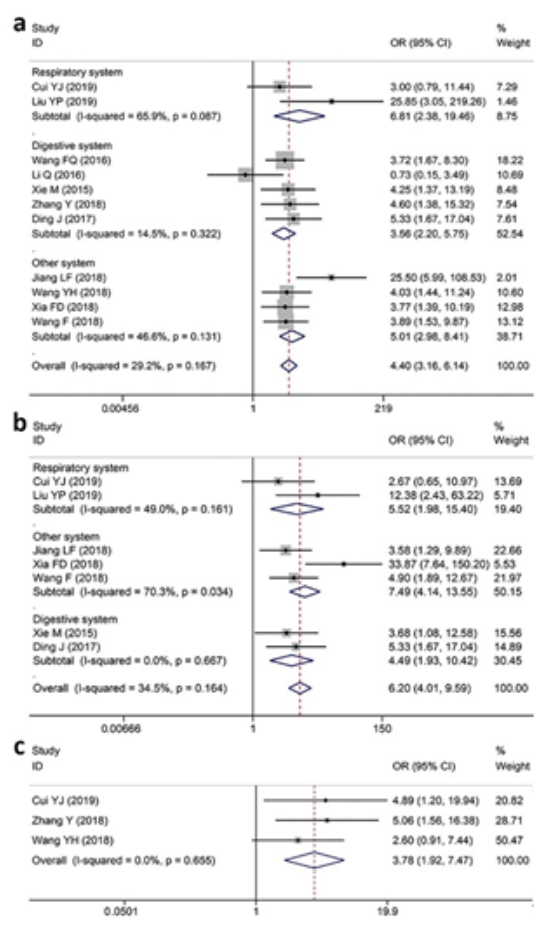

\section{Figure 3}

Forest plots evaluating the relationship between HOXA-AS2 expression and clinicopathologic features. (A) Clinical stage. (B) Lymph node metastasis. (C) Distant metastasis.

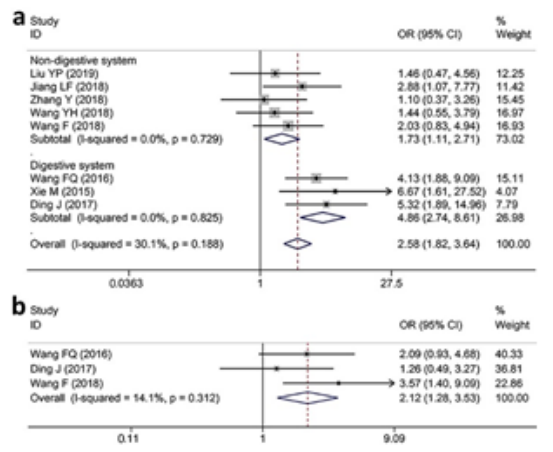

\section{Figure 4}

Forest plots evaluating the relationship between HOXA-AS2 expression and others clinicopathologic features. (A) tumor size. (B) depth of invasion. 

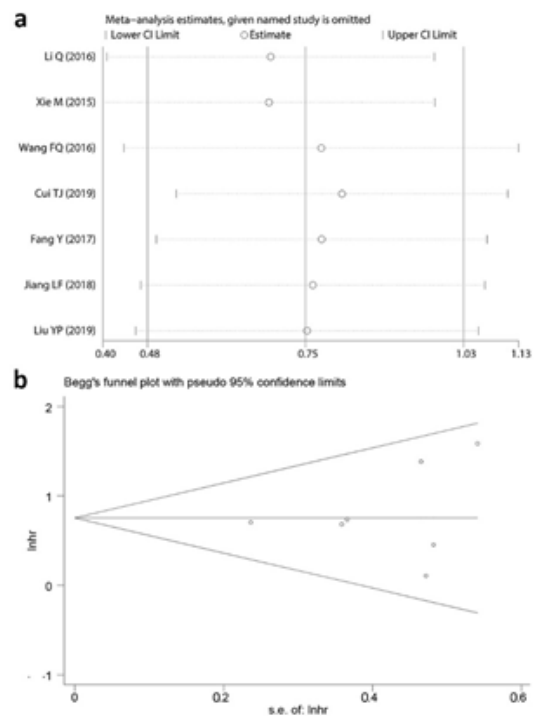

\section{Figure 5}

(A) Sensitivity analysis of pooled HR for overall survival. (B) Begg's funnel plot of HOXA-AS2 for overall survival.
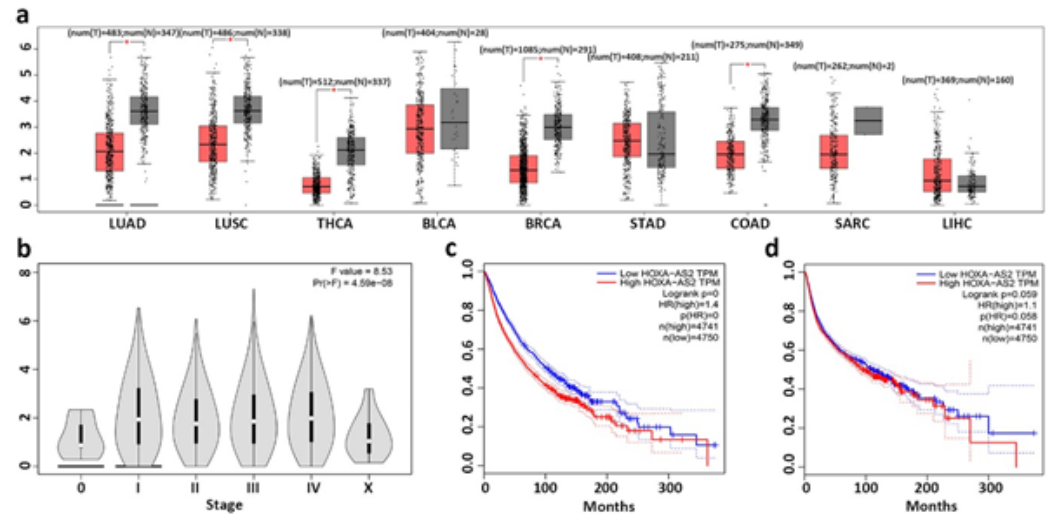

Figure 6

Validation of HOXA-AS2 expression in various cancers in GEPIA. (A) Lung adenocarcinoma (LUAD), lung squamous cell carcinoma (LUSC), thyroid carcinoma (THCA), bladder cancer (BLCA), breast invasive carcinoma (BRCA), colon adenocarcinoma (COAD), osteosarcoma (SARC), liver cancer (LIHC) and gastric cancer (STAD). (B) Violin plot showing HOXA-AS2 expression in different major clinical stage of pan-cancers in GEPIA cohort. (C) Overall survival plot of HOXA-AS2 in GEPIA cohort ( $n=9491)$. (D) Disease-free survival plot of HOXA-AS2 in GEPIA cohort $(n=9491)$.

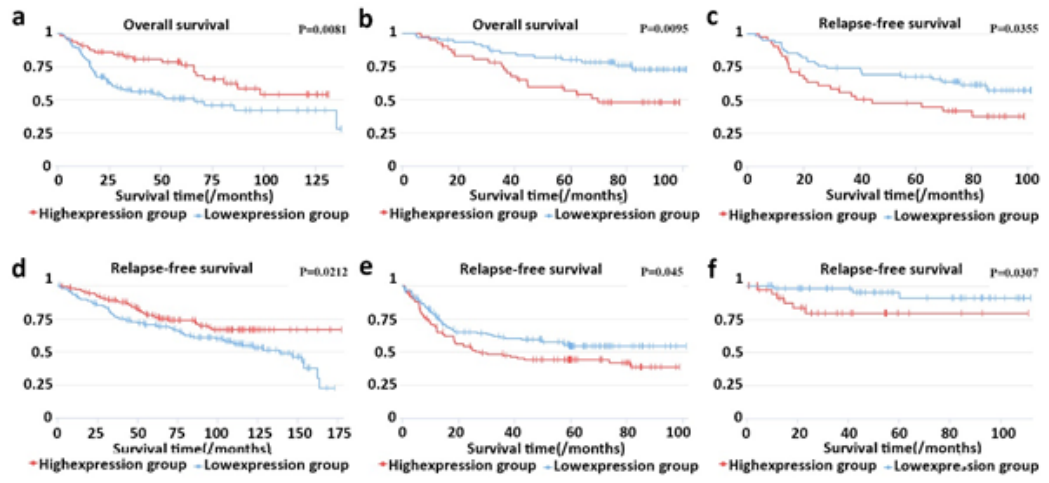

\section{Figure 7}

Survival analysis of HOXA-AS2 expression in multiple tumors. (A) Bladder cancer (B-D) Breast cancer. (E) Colorectal cancer. (F) Gastric cancer. 


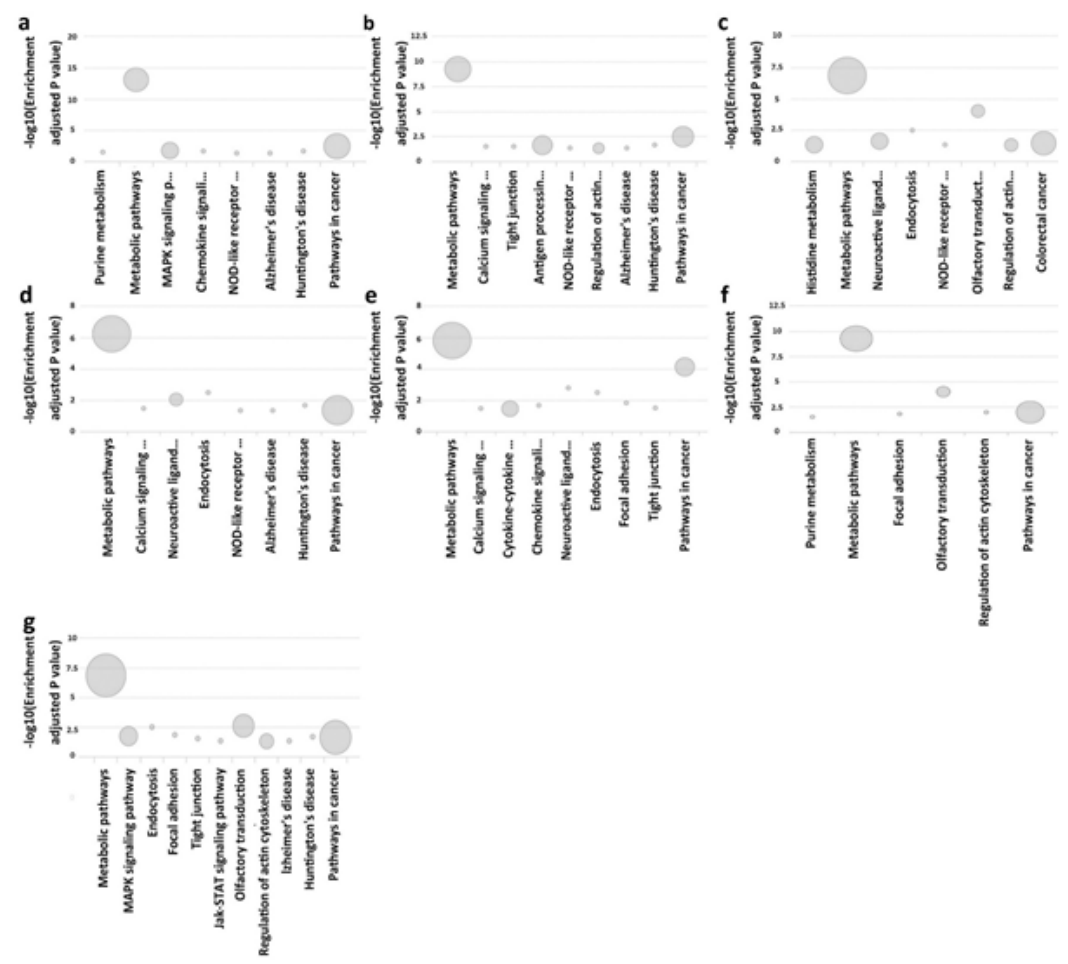

Figure 8

HOXA-AS2 correlation signaling pathway. (A) Lung cancer. (B) LUAD. (C) Large-cell lung carcinoma. (D-E) Breast cancer. (F) Colorectal cancer. (G) Hepatoblastoma.

\section{Supplementary Files}

This is a list of supplementary files associated with this preprint. Click to download.

- FigureS1.jpg 